\title{
PERFIL EPIDEMIOLÓGICO DA POPULAÇÃO PRIVADA DE LIBERDADE NOTIFICADA COM TUBERCULOSE NO TOCANTINS DE 2008 A 2018
}

\author{
EPIDEMIOLOGICAL PROFILE OF THE POPULATION DEPRIVED OF \\ LIBERTY NOTIFIED WITH TUBERCULOSIS IN TOCANTINS FROM \\ 2008 TO 2018
}

Vinícius Rosendo Vieira Campos ${ }^{1}$, Jéssica Azeredo de Carvalho², Michelle de Jesus Pantoja Filgueira ${ }^{3}$

\section{6ิ ACESSO LIVRE}

Citação: Campos VRV, Carvalho JÁ, Filgueira MJP (2021) Perfil epidemiológico da população privada de liberdade notificada com tuberculose no Tocantins de 2008 a 2018. Revista de Patologia do Tocantins, 8(1).

Instituição: ${ }^{1}$ Graduando em Medicina pela Universidade Federal do Tocantins. ${ }^{2}$ Graduanda em Medicina pela Universidade Federal do Tocantins. ${ }^{3}$ Mestre em Ensino em Ciências da Saúde pela Universidade Federal de São Paulo. 1,2,3 Universidade Federal do Tocantins, Palmas / Tocantins, Brasil.

Autor correspondente: Vinícius Rosendo Vieira Campos. Endereço: Avenida Infante Dom Henrique, 436, Tambaú, João Pessoa / Paraíba - 58039-151, Brasil. E-mail: viniciusrvcampos96@gmail.com

Editor: Carvalho A. A. B. Medicina, Universidade Federal do Tocantins, Brasil.

Publicado: 12 de maio de 2021.

Direitos Autorais: (C) 2021 Campos et al. Este é um artigo de acesso aberto que permite o uso, a distribuição e a reprodução sem restrições em qualquer meio, desde que o autor original e a fonte sejam creditados.

Conflito de interesses: os autores declararam que não existem conflitos de interesses.

\begin{abstract}
Introduction: Tuberculosis consists of an age-old infectious disease, the incidence of which is greater in vulnerable segments of society, such as the prison population. The work in question is a cross-sectional, descriptive retrospective study, which aims to identify the epidemiological profile of convicted cases notified with the disease from 2008 to 2018, in the state of Tocantins. Method: Data from the Notifiable Diseases Information System (SINAN) were used, produced from compulsory notification forms filled out by health professionals. The variables analyzed were: sex, age group, race, smoking, HIV, form of illness and situation of closure. Results: There were 61 patients, mostly men, between 25 and 34 years old, of mixed race. Smoking and HIV were not important risk factors. The pulmonary form was predominant, with 57 (93.4\%) cases, and the most common outcome was cure, totaling $39(63.9 \%)$ cases. Conclusions: Progress on the situation of tuberculosis in state prisons requires changing housing circumstances and increasing the percentage of cure among detainees. Keywords: Tuberculosis; Prisoners; Health Profile.
\end{abstract}

\section{interess.}

\begin{abstract}
RESUMO
incidênça. A tuberculose consiste em uma doença infecciosa milenar, cuja carcerária. $O$ trabalho em questão se trata de um estudo transversal retrospectivo descritivo, o qual visa a identificar o perfil epidemiológico dos casos de apenados Método: Foram utilizados dados do Sistema de Informação de Agravos de prifição (SINAN), produzidos a partir de formulários de notificação compulsória etária, raça, tabagismo, HIV, forma de doença e situação de encerramento. Resultados: Contabilizaram-se 61 pacientes, em sua maioria homens, entre 25 e 34 importantes. A forma pulmonar foi predominante, com $57(93,4 \%)$ casos, e o desfecho mais comum foi cura, somando 39 (63,9\%) casos. Conclusões: O progresso das circunstâncias de habitação e crescimento do percentual de cura entre os detentos.

Palavras-chave: Tuberculose; População Privada de Liberdade; Perfil
\end{abstract}




\section{INTRODUÇÃO}

A tuberculose consiste em uma doença infecciosa milenar, a qual acomete a humanidade desde a pré-história ${ }^{1}$ e, até hoje, permanece como uma moléstia global, chegando a afetar cerca de 10 milhões de pessoas e estando entre as dez maiores causas de morte no planeta ${ }^{2}$. Causada pelo Mycobacterium tuberculosis, pode acometer diversas estruturas do corpo, principalmente os pulmões, sendo transmitida pelas vias respiratórias de indivíduos com as formas pulmonar ou laríngea da patologia, através da eliminação de bacilos por emissão de aerossóis provenientes de tosse, fala ou espirro ${ }^{3,4}$. Vários fatores são importantes para o contágio, entre os quais as condições ambientais. Assim, devido a viver aglomerada e em locais pouco aerados e iluminados, com baixos padrões de higiene e de limpeza, além de pouco acesso a serviços de saúde, a população privada de liberdade torna-se mais vulnerável à infecção, por isso a importância de se atentar a tal grupo ${ }^{5}$.

Nesse âmbito, de acordo com o último levantamento do banco de dados "World Prison Brief", o número de indivíduos mantidos em instituições penais é maior do que 10 milhões, contabilizando para esse total tanto os prisioneiros que aguardam julgamento quanto os que cumprem sentença ${ }^{6}$. 0 Brasil, por sua vez, registrou mais de 740 mil detentos, sendo o terceiro país com a maior população carcerária do mundo, atrás apenas dos Estados Unidos e da China. Considerando que a capacidade do sistema prisional do país é de cerca de $420 \mathrm{mil}$ pessoas e que o índice de encarceramento vem crescendo sistematicamente desde o ano 2000, evidencia-se a superlotação das penitenciárias nacionais, a qual favorece a persistência da tuberculose em tal contingente e resulta no comprometimento da saúde pública.

Com efeito, estima-se que a incidência de tuberculose na população brasileira em geral é de 33 casos a cada 100 mil habitantes, enquanto que, entre os detentos, esse indicador é de 932 casos $^{7}$. Então, o risco de adoecimento por tuberculose é 28 vezes maior entre as pessoas privadas de liberdade, quando comparadas à massa em geral ${ }^{4}$. Diante disso, a fim de elucidar a tuberculose nas cadeias tocantinenses, ainda pouco exploradas, o estudo em questão tem como objetivo investigar as características sociodemográficas e clínicas dos portadores da doença entre as pessoas privadas de liberdade e identificar o perfil epidemiológico dos casos notificados no período de 2008 a 2018 no estado do Tocantins.

\section{METODOLOGIA}

\section{Coleta de Dados}

Este trabalho consistiu em uma pesquisa longitudinal, qualitativa e quantitativa, baseada em dados fornecidos por instituições governamentais (DATA-SUS ${ }^{18}$, IBGE $^{19 a, b}$, e ANS $^{20}$ ) para cada município do Tocantins, com respectivas macrorregiões de saúde, entre os anos 2008 e 2017. Os dados coletados do DATA-SUS ${ }^{18}$ foram: número absoluto de óbitos, totais (de todas as doenças somadas) e relacionados à Doença de Alzheimer (a cada ano, para ambos os sexos, em todas as faixas etárias e registrados conforme município de residência); número de médicos, total (somando-se todas as especialidades) e de especialistas capacitados ao diagnóstico da DALZ (geriatras, psiquiatras, neurologistas e neurofisiologistas); número de agentes comunitários de saúde (ACS); número de Equipes de Saúde da Família (ESF); e quantidade de aparelhos de ressonância magnética. Os dados registrados mensalmente pelo DATA-SUS foram coletados adotando-se como referência os registros de janeiro de cada ano. Já as informações referentes à população dos municípios foram obtidas conforme estimativas do IBGE ${ }^{19 a, b}$. A informação acerca da população beneficiária de Planos de Saúde no Tocantins foi acessada via ANS ${ }^{20}$, tomando-se como parâmetro o registro de informações do mês de março de cada ano.

\section{Análise de Dados}

Uma vez que o estudo fidedigno da prevalência de demências demandaria gastos exorbitantes para cobertura de amostras representativas de toda uma região ampla como os estados brasileiros (incluindo preparo técnico para padronização de critérios diagnósticos e traslados de profissionais), a prevalência da DALZ é comumente pautada em suposições baseadas em dados restritos e limitados a profissionais interessados e suas áreas de atuação, carecendo de uma cobertura mais ampla. Desta forma, mesmo que na prática a prevalência da DALZ só possa ser estimada, torna-se necessária a obtenção de dados sólidos a nível populacional a partir dos quais possam ser feitas tais projeções. Logo, propõese no presente trabalho uma nova forma de obtenção dessa informação, que chamaremos de Prevalência Indireta de Alzheimer (PIA), que consiste no seguinte:

Os óbitos referentes aos portadores da DALZ foram coletados conforme registros da categoria CID-10: G30, sendo considerado Óbito de Alzheimer Senil (OALZs) os indivíduos falecidos com mais de 65 anos e Óbito de Alzheimer Precoce (OALZp) os indivíduos falecidos com menos de 65 anos. Uma vez que a DALZ não é por si considerada uma doença letal, os registros de óbito por G30 são incorretos (provavelmente decorrentes de imprecisão da causa de morte); entretanto, podem ser utilizados como dados para um cálculo indireto da prevalência da doença, apresentado a seguir, que denominamos Prevalência Indireta. O cálculo de Prevalência Indireta consiste na obtenção de dados da prevalência de determinada patologia por meio de registros indiretos de sua existência - no caso, os registros de Alzheimer como causa mortis, mesmo que tal doença não seja causa da morte. O cálculo realizado para Prevalência Indireta é:

Prevalência Indireta de Alzheimer

$$
=\frac{O A L Z s}{\text { Número de Óbitos acima de } 65 \text { anos }}
$$

Trata-se de um estudo transversal descritivo retrospectivo referente aos casos notificados com tuberculose na população carcerária tocantinense no período de 2008 a 2018, com base em dados do Sistema de Informação de Agravos de Notificação (SINAN) $)^{8}$, produzidos a partir de formulários de notificação compulsória preenchidos por profissionais de saúde.

As variáveis epidemiológicas analisadas foram: sexo, faixa etária, raça, tabagismo, HIV, forma de tuberculose 
(pulmonar, extrapulmonar ou pulmonar e extrapulmonar) e situação de encerramento.

A determinação de médias, frequências absolutas e relativas possibilitou a análise exploratória das informações. Para organização de resultados, utilizou-se estatística descritiva simples, com a elaboração de tabelas e gráficos, por meio do software Microsoft Office Excel 2016.

\section{RESULTADOS}

De 2008 a 2018 no Tocantins foram notificados 2.287 casos de tuberculose, dos quais 61 correpondem à população privada de liberdade, 788 à população não privada de liberdade e 1.438 a casos não identificados (Tabela 1$)^{8}$. Nesse período, a média de casos por ano foi de 208,45 e o desvio padrão, 19,35, sendo 2018 o ano em que houve a maior quantidade de casos notificados.

Tabela 1: Número de casos notificados de tuberculose por ano no Tocantins de 2008 a 2018.

\begin{tabular}{|l|c|c|c|c|}
\hline $\begin{array}{l}\text { Ano } \\
\text { notificação }\end{array}$ & de & $\begin{array}{l}\text { Não } \\
\text { PPL }\end{array}$ & $\begin{array}{l}\text { Não } \\
\text { identificados }\end{array}$ & Total \\
\hline $\mathbf{2 0 0 8}$ & 0 & 0 & 197 & 197 \\
\hline $\mathbf{2 0 0 9}$ & 0 & 0 & 232 & 232 \\
\hline $\mathbf{2 0 1 0}$ & 0 & 0 & 215 & 215 \\
\hline $\mathbf{2 0 1 1}$ & 1 & 0 & 227 & 228 \\
\hline $\mathbf{2 0 1 2}$ & 0 & 1 & 202 & 203 \\
\hline $\mathbf{2 0 1 3}$ & 1 & 13 & 187 & 201 \\
\hline $\mathbf{2 0 1 4}$ & 2 & 50 & 142 & 194 \\
\hline $\mathbf{2 0 1 5}$ & 10 & 157 & 15 & 182 \\
\hline $\mathbf{2 0 1 6}$ & 13 & 186 & 12 & 211 \\
\hline $\mathbf{2 0 1 7}$ & 11 & 169 & 7 & 187 \\
\hline $\mathbf{2 0 1 8}$ & 23 & 212 & 2 & 237 \\
\hline Total & 61 & 788 & 1438 & 2287 \\
\hline
\end{tabular}

Fonte: Ministério da Saúde/SVS - Sistema de Informação de Agravos de Notificação - Sinan Net

Quando comparado à região Norte e ao Brasil, o Tocantins apresenta distinta evolução (Tabela 2 ) $^{8}$. Enquanto houve um crescimento do número de notificações nos últimos cinco anos do período estudado tanto na macrorregião referida quanto no país em geral, o estado não apresentou aumento ou mesmo redução regular da quantidade de notificações de tuberculose. Não obstante tal intermitência, o coeficiente de incidência de tuberculose (por 100 mil habitantes) do estado permaneceu abaixo das médias regional e nacional, durante esses $\operatorname{anos}^{2,9,10,11,12}$.

Tabela 2: Número de casos notificados de tuberculose por ano no Tocantins, na região Norte e no Brasil de 2008 a 2018.

\begin{tabular}{|l|c|c|c|}
\hline Ano de notificação & Tocantins & Região Norte & Brasil \\
\hline 2008 & 197 & 7828 & 83480 \\
\hline 2009 & 232 & 8328 & 86475 \\
\hline 2010 & 215 & 8357 & 85281 \\
\hline 2011 & 228 & 8768 & 87602 \\
\hline 2012 & 203 & 8556 & 86114 \\
\hline 2013 & 201 & 8888 & 86841 \\
\hline
\end{tabular}

\begin{tabular}{|l|c|c|c|}
\hline 2014 & 194 & 8752 & 85007 \\
\hline $\mathbf{2 0 1 5}$ & 182 & 9008 & 85361 \\
\hline $\mathbf{2 0 1 6}$ & 211 & 9357 & 85706 \\
\hline $\mathbf{2 0 1 7}$ & 187 & 10178 & 90485 \\
\hline $\mathbf{2 0 1 8}$ & 237 & 10437 & 94166 \\
\hline
\end{tabular}

Fonte: Ministério da Saúde/SVS - Sistema de Informação de Agravos de Notificação - Sinan Net

Em se tratando especificamente do âmbito prisional, no entanto, percebe-se que os casos notificados no Tocantins apresentaram sim uma tendência de elevação ao longo do tempo. Do total de 61 notificações correspondentes à população privada de liberdade, 59 (96,7\%) ocorreram na segunda metade da década.

Sobre as variáveis, com relação ao sexo (Tabela 3$)^{8}$, em todos os anos analisados, as notificações de pacientes masculinos foram superiores ou iguais à de pacientes femininos. Dos casos apurados, 55 (90,2\%) eram de homens e $6(9,8 \%)$ de mulheres. Válido apontar que não houve notificações femininas no biênio 2016-2017, apesar do registro de casos nos dois anos anteriores. Em contraste, houve tendência de aumento de notificações masculinas, a partir de 2014.

Quanto à idade (Tabela 3), as faixas etárias mais jovens contabilizaram a maioria das notificações, enquanto os pacientes acima de 45 anos somaram apenas 10 casos (16,3\%). Observou-se maior prevalência na faixa de 25 a 34 anos, com 22 pacientes (36\%). Não houve formulários com esse campo ignorado ou em branco.

No que diz respeito à raça (Tabela 3), em geral, a prevalência das notificações seguiu a distribuição étnica do estado ${ }^{13}$, sendo o grupo pardo o mais acometido, com 38 pacientes (62\%). A exceção foi o indígena, que, embora corresponda ao menor grupo étnico do Tocantins e das penitenciárias estaduais ${ }^{14}$, correspondeu ao segundo mais notificado, juntamente ao branco, cada um com 8 casos (13\%).

Tabela 3: Distribuição dos casos por sexo, faixa etária e raça.

\begin{tabular}{ccc}
\hline $\begin{array}{c}\text { Indicadores } \\
\text { Sexo }\end{array}$ & Frequência absoluta & Frequência relativa \\
\hline Masculino & 55 & $90,2 \%$ \\
\hline Feminino & 6 & $9,8 \%$ \\
\hline
\end{tabular}

\begin{tabular}{|ccc|}
\hline Faixa Etária & & \\
\hline $\mathbf{1 5 - 2 4}$ & 15 & $24,6 \%$ \\
\hline $\mathbf{2 5 - 3 4}$ & 22 & $36,1 \%$ \\
\hline $\mathbf{3 5 - 4 4}$ & 14 & $22,9 \%$ \\
\hline $\mathbf{4 5 - 5 4}$ & 4 & $6,6 \%$ \\
\hline $\mathbf{5 5 - 6 4}$ & 3 & $4,9 \%$ \\
\hline $\mathbf{6 5}$ ou mais & 3 & $4,9 \%$ \\
\hline
\end{tabular}

\begin{tabular}{ccc}
\hline Raça & & \\
\hline Branca & 8 & $13,1 \%$ \\
\hline Preta & 4 & $6,6 \%$ \\
\hline Amarela & 1 & $1,6 \%$ \\
\hline Parda & 38 & $62,3 \%$ \\
\hline Indígena & 8 & $13,1 \%$ \\
\hline Ignorado & 2 & $3,3 \%$ \\
\hline Total & 61 & $100 \%$ \\
\hline
\end{tabular}

Fonte: Ministério da Saúde/SVS - Sistema de Informação de Agravos de Notificação - Sinan Net 
Fonte: Ministério da Saúde/SVS - Sistema de Informação de

Já os resultados de tabagismo (Tabela 4$)^{8}$ apontaram que a maioria das notificações no período estudado foi de pacientes não fumantes. Esses constituíram 35 casos (57,3\%) - mais do que o dobro de notificações de pacientes fumantes, que totalizaram 15 casos $(24,6 \%)$. Onze casos foram registrados como "ignorado/branco".

Acerca do HIV (Tabela 4), averiguou-se que a maioria dos casos foi identificada como não portadora. Dos 52 pacientes testados, 50 (81,9\%) foram negativos para o vírus. Destaca-se também uma quantidade pertinente de exames não realizados - $9(14,7 \%)$ entre o total de notificações.

Tabela 4: Distribuição dos casos por tabagismo e HIV.

\begin{tabular}{lcc}
\hline Indicador & Frequência absoluta & Frequência relativa \\
\hline Tabagismo & & \\
\hline Sim & 15 & $24,6 \%$ \\
\hline Não & 35 & $57,3 \%$ \\
\hline Ignorado & 11 & $18,1 \%$ \\
\hline & & \\
\hline HIV & & $3,3 \%$ \\
\hline Positivo & 2 & $81,9 \%$ \\
\hline Negativo & 50 & $14,8 \%$ \\
\hline Não realizado & 9 & $100 \%$ \\
\hline Total & 61 & \\
\hline
\end{tabular}

Fonte: Ministério da Saúde/SVS - Sistema de Informação de Agravos de Notificação - Sinan Net

No que toca à forma de doença (Tabela 5$)^{8}$, a tuberculose pulmonar foi a mais prevalente, com 57 (86,3\%) notificações. Dos casos extrapulmonares, 1 foi pleural, em 2015; todos os demais foram de gânglios periféricos, em 2011, 2016 e 2017. Não houve registro de acometimento pulmonar e extrapulmonar simultâneo.

Por fim, a situação de encerramento (Tabela 5) apontou que 39 $(63,9 \%)$ pacientes tiveram alta por cura, 11 (18,0\%) abandonaram o tratamento, seja de forma primária ou não, 3 $(4,9 \%)$ foram transferidos de unidade prisional e $2(3,2 \%)$ vieram a óbito - desses apenas 1 consequente à tuberculose. Os casos resistentes a medicamentos contabilizaram $2(3,2 \%)$, e houve $4(6,5 \%)$ notificações sem informação preenchida.

Tabela 5: Distribuição de casos por forma de doença e situação de encerramento.

\begin{tabular}{|c|c|c|c|}
\hline Indicadores & & $\begin{array}{l}\text { Frequência } \\
\text { absoluta }\end{array}$ & $\begin{array}{l}\text { Frequência } \\
\text { relativa }\end{array}$ \\
\hline \multicolumn{4}{|l|}{ Forma de doença } \\
\hline Pulmonar & & 57 & $93,4 \%$ \\
\hline Extrapulmonar & & 4 & $6,6 \%$ \\
\hline $\begin{array}{l}\text { Pulmonar } \\
\text { Extrapulmonar }\end{array}$ & e & 0 & $0 \%$ \\
\hline
\end{tabular}

Situação de

Encerramento

\begin{tabular}{lcc}
\hline Cura & 39 & $63,9 \%$ \\
\hline Abandono & 11 & $18,0 \%$ \\
\hline Óbito & 2 & $3,3 \%$ \\
\hline Transferência & 3 & $4,9 \%$ \\
\hline TB-DR & 2 & $3,3 \%$ \\
\hline Ignorado & 4 & $6,6 \%$ \\
\hline Total & 61 & $100 \%$ \\
\hline
\end{tabular}

Agravos de Notificação - Sinan Net

\section{DISCUSSÃO}

Verifica-se que houve pouca variação do número de notificações de tuberculose no Tocantins ao longo do tempo. Uma concebível explicação seria carência ou ineficiência de intervenções públicas para modificação desse panorama. A significativa porção de casos não identificados soma para a possibilidade de negligência de múltiplos setores de gestão, seja no preenchimento de formulários de notificação, seja na elaboração de políticas de saúde adequadas. Embora tenha reduzido consideravelmente a partir de 2015, tal falha dificulta o legítimo entendimento da evolução da tuberculose no estado.

O crescimento da quantidade de casos observado na região norte e no país em geral, bem como no contingente encarcerado no Tocantins, por sua vez, não é necessariamente adverso. Não obstante possa sugerir aumento da incidência de casos, pode igualmente indicar avanço no sistema de notificações, sendo esta hipótese corroborada pelo melhor preenchimento dos formulários nos anos mais recentes do período estudado.

Considerando a população tocantinense detenta notificada com tuberculose, quanto ao sexo, o maior número de notificações foi de pacientes homens. Tal resultado não surpreende, visto que é produto da realidade das cadeias masculinas do estado, cujo número de apenados é sobremaneira superior à das cadeias femininas, bem como o excesso de contingente ${ }^{13}$. Portanto, maior a aglomeração, maior o risco de contrair tuberculose.

A idade averiguada é comum a outras localidades. Pesquisas realizadas em penitenciárias do Espírito Santo ${ }^{5}$, de São Paulo ${ }^{15}$, do Ceará ${ }^{16}$ também apuraram que a maior parte dos pacientes de tuberculose constatados pertenciam a faixa etária em torno de 25 a 34 anos. Assim, considera-se que o resultado não apenas é um reflexo da população encarcerada do estado, como também do país.

Em referência ao observado para etnia, poder-se-ia pensar que tal fenômeno está relacionado a uma possível omissão do sistema penitenciário estatal com o grupo indígena, colocandoo em condições mais susceptíveis de contrair a doença. Contudo, dado que metade desses casos ocorreu no mesmo ano (2015) e que inexistem evidências de destrato de indígenas nas cadeias estatais, não há sustentação para essa hipótese, sendo mais provável que o resultado encontrado se deve a um contato maior entre indivíduos que viviam em proximidade. No tocante a tabagismo, é de conhecimento que seja um fator de risco para infecção, doença e mortalidade por tuberculose ${ }^{17}$. Com efeito, a fumaça do cigarro está associada à disfunção ciliar, redução da resposta imune, falha na atividade de macrófagos e alteração dos níveis de $\mathrm{CD} 4^{18}$. Na população privada de liberdade tocantinense, entretanto, esse não se constituiu em um elemento significativo para contrair a doença. Tal qual tabagismo, a associação entre HIV e tuberculose é notável. Estima-se que cerca de um terço do total de soropositivos do mundo seja igualmente infectada pelo bacilo 
de Koch $^{19}$. Ademais, como os presidiários vivem em condições favoráveis para a propagação do vírus ${ }^{20}$, é necessário avaliar se essa relação é relevante para o Tocantins. Todavia o HIV não se mostrou como um fator de risco importante nesse contexto.

A forma pulmonar da doença, por sua vez, acometeu a maioria dos pacientes. O Mycobacterium tuberculosis tem predileção por ambientes aerófilos, pois se trata de uma bactéria aeróbia estrita ${ }^{21}$. Por conseguinte, o acometimento pulmonar é o mais comum, uma vez que os pulmões são altamente oxigenados. Essa constatação não só é condizente nas cadeias, como ainda é agravada, visto que as condições do cárcere tornam o contato com o bacilo ainda mais recorrente. Relativamente à situação de encerramento, considera-se que, o controle da tuberculose no sistema carcerário tocantinense não está sendo eficiente. Apesar de cura ter sido o desfecho mais prevalente, o índice de abandono foi muito alto, totalizando quase $20 \%$. As frequências de casos transferidos e ignorados também foram significativas, dificultando o controle de evolução da terapia. Destarte, a soma de todos esses fatores favorece a resistência às terapias medicamentosas e a disseminação da doença.

\section{CONCLUSÃO}

Tem-se, pois, a exposição de alguns aspectos referentes à população privada de liberdade do Tocantins notificada com tuberculose. Ainda que não seja admissível afirmar se foi feita de fato a análise de todos os casos ao longo de uma década, tendo em vista a grande quantidade de formulários não identificados, discorreu-se acerca do que foi devidamente notificado, estabelecendo o perfil desses pacientes.

Assim sendo, em relação às características sociodemográficas, percebeu-se que tais indivíduos, em geral, refletem a população encarcerada do estado, a qual é, em sua maioria, masculina, jovem e parda. Já as avaliações de tabagismo e de HIV foram particularmente relevantes, sobretudo para o norteamento de políticas de intervenção efetivas, que envolvam ações educativas e integradas dos profissionais de saúde e de segurança, a fim de elucidar a tuberculose nas cadeias, diminuir os preconceitos associados à doença e evidenciar o problema.

A prevalência da forma pulmonar da patologia, outrossim, mais do que reafirmar a epidemiologia da doença, evoca a necessidade de melhorar as condições que contribuem para sua disseminação nas cadeias: superlotação, pouca iluminação, baixa ventilação. Enfim, um fator agravante que igualmente urge solução foi o percentual de abandono de tratamento, sustentando o contágio entre indivíduos. Assim, é preciso orientar adequadamente o paciente e criar mecanismos que permitam o acompanhamento regular, não só no período crítico de infecção, mas também ao longo de toda a duração da terapia.

\section{REFERÊNCIAS BIBLIOGRÁFICAS}

1. Daniel TM. The history of tuberculosis. Respiratory Medicine, [s.I.], v. 100, n. 11, p.1862-1870, nov. 2006. Elsevier BV.

http://dx.doi.org/10.1016/j.rmed.2006.08.006.

2. Brasil. Ministério da Saúde. Secretaria de Vigilância em Saúde. Boletim Epidemiológico. 50 v. (9) [Internet]. 2019 [acessado em 23 jan. 2020]. Disponível em: https://portalarquivos2.saude.gov.br/images/pdf/2019/ marco/22/2019-009.pdf

3. Melo FAF, Klautau GB, Rodrigues DSS, Afiune JB, Hijjar MA, Gomes M. Tuberculose; et al. In: Veronesi R, Focaccia R. Tratado de Infectologia. 5. ed. São Paulo: Atheneu, 2015. Cap. 66. p. 1399-1470.

4. Brasil. Ministério da Saúde. Secretaria de Vigilância em Saúde (org.). Manual de Recomendações para o controle da Tuberculose no Brasil. 2. ed. [Internet]. 2019 [acessado em 23 jan. 2020]. Disponível em: http://bvsms.saude.gov.br/bvs/publicacoes/manual_reco mendacoes_controle_tuberculose_brasil_2_ed.pdf

5. Macedo LR, Macedo CR, Maciel ELN. Vigilância Epidemiológica da Turberculose em Presídios do Espírito Santo. Revista Brasileira em Promoção da Saúde, [s.I.], v. 26, n. 2, p. 216-222, 30 jun. 2013. Fundação Edson Queiroz. http://dx.doi.org/10.5020/18061230.2013.p216

6. Institute for Crime \& Justice Policy Research. World Prison Brief [Internet]. 2018 [acessado em 23 jan. 2020]. Disponível em: https://www.prisonstudies.org/

7. Brasil. Ministério da Saúde. Tuberculose: o que é, causas, sintomas, tratamento, diagnóstico e prevenção.

[Internet]. 2020 [acessado em 23 jan. 2020]. Disponível em: https://www.saude.gov.br/

8. Brasil. Ministério da Saúde. DATASUS [Internet]. 2020 [acessado em 23 jan. 2020]. Disponível em: http://datasus.saude.gov.br/informacoes-de-saudetabnet/

9. Brasil. Ministério da Saúde. Secretaria de Vigilância em Saúde (org.). Boletim Epidemiológico. 49 v. (11) [Internet]. 2018 [acessado em 23 jan. 2020]. Disponível em:

https://portalarquivos2.saude.gov.br/images/pdf/2018/ marco/26/2018-009.pdf

10. Brasil. Ministério da Saúde. Secretaria de Vigilância em Saúde (org.). Boletim Epidemiológico. 48 v. [Internet]. 2017 [acessado em 23 jan. 2020]. (8). Disponível em: https://portalarquivos2.saude.gov.br/images/pdf/2017/ marco/23/2017-V-48-N-8-Indicadores-priorit--rios-parao-monitoramento-do-Plano-Nacional-pelo-Fim-daTuberculose-como-Problema-de-Sa--de-P--blica-noBrasil.pdf

11. Brasil. Ministério da Saúde. Secretaria de Vigilância em Saúde (org.). Boletim Epidemiológico. 47 v. (13) [Internet]. 2016 [acessado em 23 jan. 2020]. Disponível em:

<http://portalarquivos.saude.gov.br/images/pdf/2016/m arco/24/2016-009-Tuberculose-001.pdf>.

12. Brasil. Ministério da Saúde. Secretaria de Vigilância em Saúde (org.). Boletim Epidemiológico. 46 v. (9) [Internet]. 
2015 [acessado em 23 jan. 2020]. Disponível em: http://portalarquivos.saude.gov.br/images/pdf/2015/ma rco/25/Boletim-tuberculose-2015.pdf

13. Instituto Brasileiro de Geografia e Estatística. Censo Demográfico 2010 [Internet]. 2010 [acessado em 25 fev. 2020]. Disponível em: https://sidra.ibge.gov.br/home/ipca/brasil

14. Brasil. Ministério da Justiça e da Segurança Pública. Departamento Penitenciário Nacional. Infopen [Internet]. 2020 [acessado em 25 fev. 2020]. Disponível em: http://depen.gov.br/DEPEN/depen/sisdepen/infopen/rel atorios-analiticos/TO/to

15. Oliveira HB, Cardoso JC. Tuberculose no sistema prisional de Campinas, São Paulo, Brasil. Revista Panamericana de Salud Pública, São Paulo, v. 15, n. 3, p. 194-199. [Internet] 2004 [acessado em 25 fev. 2020]. Disponível em: https://www.scielosp.org/article/rpsp/2004.v15n3/194199/\#ModalArticles.

16. Gouveia GPM, Gouveia SSV, Bezerra Filho JG, Oliveira JBB. Estudo epidemiológico da tuberculose pulmonar no Hospital Penitenciário e Sanatório Professor Otávio Lobo no período de 2001-2006. Revista Baiana de Saúde Pública, [S. L.], v. 34, n. 3, p. 602-611, jul./set. 2010. https://doi.org/10.22278/2318-2660.2010.v34.n3.a59

17. Rabahi MF. Tuberculose e Tabagismo. Pulmão Rj, Rio de Janeiro, v. 21, n. 1, p. 46-49. [Internet] 2012 [acessado em 21 mar. 2020]. Disponível em: http://www.sopterj.com.br/wpcontent/themes/_sopterj_redesign_2017/_revista/2012/ n_01/11.pdf

18. Silva DR, Muñoz-Torrico $M$, Duarte $R$, Galvão $T$, Bonini EH, Arbex FF, Arbex MA, Augusto VM, Rabahi MF, Mello FCQ. Risk factors for tuberculosis: diabetes, smoking, alcohol use, and the use of other drugs. Jornal Brasileiro de Pneumologia, [S.L.], v. 44, n. 2, p. 145-152, abr. 2018. FapUNIFESP (SciELO). http://dx.doi.org/10.1590/s180637562017000000443.

19. Getahun H, Gunneberg C, Granich R, Nunn P. HIV Infection-Associated Tuberculosis: the epidemiology and the response. Clinical Infectious Diseases, [S.L.], v. 50, n. 3, p. 201-207, 15 maio 2010. Oxford University Press (OUP). http://dx.doi.org/10.1086/651492.

20. Coelho HC, Perdoná GC, Neves FR, Passos ADC. HIV prevalence and risk factors in a Brazilian penitentiary. Cadernos de Saúde Pública, [S.L.], v. 23, n. 9, p. 2197-2204, set. 2007. FapUNIFESP (SciELO). http://dx.doi.org/10.1590/s0102-311×2007000900027.

21. Mascarenhas MDM, Araújo LM, Gomes KRO. Perfil epidemiológico da tuberculose entre casos notificados no Município de Piripiri, Estado do Piauí, Brasil. Epidemiologia e Serviços de Saúde, [s.I.], v. 14, n. 1, p. 7-14, mar. 2005. Instituto Evandro Chagas. http://dx.doi.org/10.5123/s1679-49742005000100002. 